\title{
Cancer, Mortality, and Acute Kidney Injury Among Hospitalized Patients with SARS-CoV-2 Infection
}

\author{
Johnathan Khusid $^{1}$, Adan Z. Becerra ${ }^{2}$, Blair Gallante ${ }^{1}$, Areeba S. Sadiq ${ }^{1}$, William M. \\ Atallah $^{1}$, Ketan K. Badani ${ }^{1}$, and Mantu Gupta ${ }^{1}$ \\ ${ }^{1}$ Icahn School of Medicine at Mount Sinai \\ ${ }^{2}$ Rush University Medical Center
}

September 16, 2020

\begin{abstract}
Background: To evaluate COVID19 patients treated within our academic medical system to determine if history of malignancy, both in general and specifically in genitourinary oncology patients, is associated with adverse clinical outcomes, including AKI and mortality. Methods: We conducted a retrospective cohort study among patients with confirmed SARS-CoV-2 infection in a multi-hospital, academic medical institution in New York City. Outcomes included mortality, ICU admission and AKI among hospitalized patients. We also evaluated risk of hospitalization among all patients with SARS-CoV-2 infection. Multilevel logistic regression models were used for analysis. Results: We identified 6,893 patients who met inclusion criteria, of which 4,018 were hospitalized. Among hospitalized patients $374(9 \%)$ had a history of cancer, 281 (7\%) experienced AKI, and 1,045 $(26 \%)$ died. In adjusted analyses, patients with a history of cancer had $1.33(95 \% \mathrm{CI}=1.05,1.69)$ times the odds of death compared to those without cancer and this appeared to be driven by lung cancer $(\mathrm{OR}=2.44,95 \% \mathrm{CI}=1.05,4.39)$. Patients with a history of genitourinary cancer were not at higher risk of mortality compared to those without cancer $(\mathrm{OR}=0.99,95 \% \mathrm{CI}=$ $0.61,1.62)$. History of cancer was not associated with ICU admission or AKI in overall and subgroup analyses. Conclusions: Patients with a history of cancer who are hospitalized with SARS-CoV-2 infection are not at greater risk for AKI, though they are at higher risk for mortality as compared to patients without a history of cancer. The increased risk in mortality appears driven by patients with pulmonary neoplasms. Patients with a history of genitourinary malignancies do not appear to be at higher risk for AKI or for mortality compared to the general population.
\end{abstract}

\section{Conclusions:}

Patients with a history of cancer who are hospitalized with SARS-CoV-2 infection are not at greater risk for AKI, though they are at higher risk for mortality as compared to patients without a history of cancer. The increased risk in mortality appears driven by patients with pulmonary neoplasms. Patients with a history of genitourinary malignancies do not appear to be at higher risk for AKI or for mortality compared to the general population.

\section{What is already known about this topic?}

-Acute kidney injury is common in patients hospitalized with COVID19

-Cancer history may be associated with adverse outcomes in COVID19 patients

-Optimal kidney function is important for cancer patients as many chemotherapeutic agents are nephrotoxic

What does this article add?

- We conducted a retrospective cohort study among patients with confirmed SARS-CoV-2 infection in a multi-hospital, academic medical institution in New York City. 
-Cancer, especially lung cancer, was associated with mortality, but was not associated with acute kidney injury

-Genitourinary cancer patients had a similar risk compared to noncancer patients.

\section{MAIN TEXT}

\section{INTRODUCTION}

The Coronavirus Disease-2019 (COVID19) pandemic has led to radical shifts in the delivery of healthcare. To minimize the risk of person-to-person transmission, particularly in the nosocomial setting, attempts have been made to increase utilization of telehealth(1). Yet, many aspects of healthcare require in-person interactions. This is particularly true for urologic oncology patients as chemotherapy infusions, radiation therapy, surgical expatriation, and radiologic surveillance are components of routine care. Furthermore, the time-sensitive nature of malignancy makes delays in care problematic for many patients.

To this end, several collaborative reviews have been published on strategies for the appropriate triage of patients with urologic malignancy during the COVID19 pandemic(2-4). These recommendations are largely based on the existing literature regarding the natural history of individual urologic malignancies. For highly aggressive malignancies such as muscle invasive bladder cancer, significant delays in care are clearly unacceptable(5). Similarly, for those malignancies with a more indolent behavior such as low-risk prostate cancer, delays and modifications to routine care are unlikely to produce significant adverse outcomes(6). However, for moderately aggressive malignancies such as intermediate-risk and high-risk prostate cancer, non-muscle invasive bladder cancer, and most kidney cancers, the risks and benefits of delaying and modifying care should be based both on the natural history of the cancer, and the natural history of COVID-19 in these patients and cancer patients in general.

Indeed, several large population-based studies from various countries have suggested malignancy is associated with adverse outcomes in COVID19 patients(7-9). Furthermore, studies from cancer centers and cancer consortiums have evaluated the natural history of COVID19 in larger cohorts of cancer patients and found a mortality rate ranging from $12 \%-28 \%(10-12)$. Interestingly, malignancy type did not appear to be a significant predictor of mortality. However, a notable limitation of the existing literature is that many studies utilized small cohorts of cancer patients or did not have a control group of patients without a cancer diagnosis. Furthermore, though our understanding of COVID19 and cancer has continued to evolve, little is known about the impact of cancer history on the risk of developing acute kidney injury (AKI) in COVID19 patients. AKI occurs in an estimated 4.5-8.9\% of COVID19 cases(13, 14). AKI increases the risk of developing CKD(15), which is concerning for cancer patients as many oncologic therapies may result in renal impairment(16). Additionally, in patients with genitourinary malignancies, CKD has been associated with adverse post-operative outcomes(17-19).

Our academic medical system includes several hospitals and outpatient clinics throughout the New York City area, which has been one of the world's most afflicted locations and in turn has treated a large population of COVID19 patients. Accordingly, in the present study, we seek to evaluate COVID19 patients treated within our academic medical system to determine if history of malignancy, both in general and specifically in genitourinary oncology patients, is associated with clinical outcomes, including AKI and mortality.

\section{MATERIALS AND METHODS}

\section{Data Source and Study Design}

Our study received Institutional Review Board Approval (HS\#: 20-00875). The study was conducted at a large, New York City based, multi-hospital, academic medical institution. The institution's Scientific Computing team has been maintaining a regularly updated, deidentified, database of patient-level data from our electronic medical record (EMR) system for encounters related to COVID19. At the time of data extraction for the present study, the database included encounters from March $1^{\text {st }}, 2020$ through August $10^{\text {th }}, 2020$. 
We included all patients who had a healthcare encounter indicating a positive result for the severe acute respiratory syndrome coronavirus 2 (SARS-CoV2) real-time reverse transcriptase-polymerase-chain-reaction (RT-PCR) assay test during the study period. We identified patient characteristics including demographics, comorbidities (including historical cancer diagnoses), and outcomes from the EMR as well as the location of the encounter. If patients had multiple encounters, an encounter with a positive result was chosen, if applicable. If patients had multiple patient encounters with a positive result, the most severe encounter was chosen. Patients with missing data on any of the variables included in the analysis were excluded.

\section{Outcomes}

The primary outcome was all-cause mortality among hospitalized patients (inpatient encounters) with SARSCoV2 infection. We also evaluated intensive care unit (ICU) admission and AKI among hospitalized patients. Further, we examined hospitalization among all encounters (inpatient and outpatient). The primary analysis compared outcomes between patients with a history of cancer to those without a history of cancer. Secondary analyses compared outcomes between patients with specific neoplasm locations (kidney, prostate, bladder, lung, hematological, general genitourinary). Oncologic co-morbidities were determined by ICD-10 codes in patients' problem lists in the EMR. Patients were included in the specific neoplasm location cohorts regardless of whether the ICD-10 noted the neoplasm as primary, secondary, or unspecified.

\section{Potential Confounders}

Because patients with a history of cancer may be different to those without a history of cancer in ways that could be related to the outcomes, the analysis accounted for a comprehensive list of potential confounders. Analyses adjusted for the following variables: age, sex, race, insurance status, smoking status, diabetes, obesity, hypertension, coronary artery disease, chronic kidney disease, atrial fibrillation, asthma, and chronic obstructive pulmonary disorder. Additionally, we performed subgroup analysis on patients with hematologic and lung malignancies given the common pulmonary and hematologic sequalae of COVID19(20).

\section{Statistical Analysis}

We used descriptive statistics and bivariate tests to characterize distributions of covariates across groups for the overall cohort and among those hospitalized. Variables associated with the outcome at a p-value $<0.20$ in bivariate tests were included in multivariable analyses. We used multilevel logistic regression models for all outcomes to account for clustering of patient outcomes among individual hospitals in the health system. Models estimated odds ratios and $95 \%$ confidence intervals (CI).

In sensitivity analyses, we analyzed all-cause mortality and hospitalization using a multilevel Cox proportional hazards model instead. Further, we analyzed AKI and ICU using a multilevel Cox proportional hazards model with mortality as a competing risk. The Cox proportional hazards assumption was verified graphically by generating a plot of the Schoenfeld residuals. In all instances, our primary results were robust and sensitivity analyses yielded similar inferences. Analyses were conducted with R, Version 4.0.0, and used 2 -sided statistical tests with a p value $<0.05$ as statistically significant in final analyses.

\section{RESULTS}

We identified 10,429 unique patients who met study inclusion criteria. Among these, we excluded 3,596 patients who had missing information leaving us with an overall sample size of 6,893 patients with an inpatient or outpatient encounter (Figure 1). Among these patients, 4,018 were hospitalized, and 720 had a history of cancer. Table 1 reports patient characteristics overall and by mortality status among hospitalized patients (primary analysis). Among the 4,018 hospitalized patients, the average age was 64, 1,844 (46\%) were female, 1,060 (26\%) were Black, $374(9 \%)$ had a history of cancer, and 1,045 (26\%) died.

Table 2 reports multivariable results for the primary and secondary analyses. For the primary analysis, patients with a history of cancer had $33 \%$ increased adjusted odds of mortality compared to patients without a history of cancer $(\mathrm{OR}=1.33,95 \% \mathrm{CI}=1.05,1.69)$. This effect appeared to be largely driven by the increased risk associated specifically with lung cancer. Patients with lung cancer had $144 \%$ increased adjusted odds 
of mortality compared to patients without a history of cancer $(\mathrm{OR}=2.44,95 \% \mathrm{CI}=1.05,4.39)$. Patients with kidney cancer, bladder, cancer, hematological cancer, and all genitourinary cancers did not have an increased mortality risk compared to patients without a history of cancer. Further, patients with prostate cancer had $71 \%$ increased adjusted odds of mortality compared to patients with other cancers $(\mathrm{OR}=1.71$, $95 \% \mathrm{CI}=1.02,2.92)$. Finally, patients with kidney cancer $(\mathrm{OR}=0.41,95 \% \mathrm{CI}=0.18,0.95)$ and those with all genitourinary cancers had lower odds of hospitalization compared to other cancers. We did not observe any other statistically significant effects.

\section{DISCUSSION}

For cancer patients, navigating the COVID19 pandemic has brought unique challenges. Balancing the risk of contracting COVID19 against the risk of delaying oncologic care has thus far been informed by the natural history of malignancies, while the natural history of COVID19 in cancer patients remains an area of active research. Prior studies have attempted to define clinical outcomes in cancer patients with COVID19 (7, $8,10-12,21)$. One of the first such studies evaluated 1590 patients from China with COVID19, of which 18 patients had a history of malignancy, and found that a history of malignancy was associated with poor clinical outcomes(8). Another population based study from Spain evaluated 4,035 consecutively hospitalized patients with COVID19 and found that presence of an active malignancy (359 patients) was an independent predictor of mortality(7). A study of 5,366 COVID19 patients in New York City early in the pandemic found that a history of cancer (334 patients) conferred a greater risk of intubation, though not a greater risk of death(9).

Studies from cancer centers have evaluated the natural history of COVID19 in larger cohorts of cancer patients. A prospective observational study of 800 cancer patients in the United Kingdom with COVID19 reported a $28 \%$ mortality rate. Interestingly, the risk of mortality was independent of cancer type(11). However, in a cohort of 423 patients treated at a cancer center New York City, the mortality rate was notably lower at only 12\%(12). Indeed, a multi-institutional study of 928 patients with COVID19 and cancer throughout the US and Canada found a mortality rate of 13\%(10). Malignancy type was not a significant predictor of mortality. Notably, none of these studies compared outcomes to those of patients without cancer. One meta-analysis which included 46,499 COVID19 patients, including 1776 of whom had cancer, found that malignancy is associated with an increased risk of death and intubation(21). However, in a subgroup analysis of patients $>65$, there was no increased risk of mortality from COVID19 amongst cancer patients.

Though our understanding of COVID19 and cancer has continued to evolve, little is known about the impact of a history of cancer on the risk of developing AKI in COVID19 patients. Indeed, to our knowledge, no such studies have evaluated AKI as an outcome. Furthermore, many of the existing studies had relatively small samples of cancer patients or did not have a non-cancer patient control. To this end, in the present study, we have a conducted a retrospective analysis of a large cohort of patients hospitalized with COVID19 in the New York City area to evaluate the impact of malignancy on patient outcomes, including AKI, with a focus on patients with genitourinary malignancies.

Notably, our cohort represents approximately $4 \%$ of positive cases in New York City and $7 \%$ of deaths in New York City as of August $10^{\text {th }}, 2020(22)$. Approximately $7 \%$ of all hospitalized COVID19 patients in our cohort had a diagnosis AKI, an incidence concordant with the 4.5\%-8.9\% range reported in the literature(13, 14). Notably, there was no increased risk of AKI amongst patients with a general history of malignancy, or amongst patients with a history of genitourinary malignancy. This key finding informs us that patients with a history of cancer are not more likely to develop AKI when hospitalized with COVID19. AKI is of particular concern for cancer patients as AKI is associated with the subsequent development of CKD, and CKD is associated with adverse post-operative outcomes in patients with prostate, kidney, and bladder cancer(1719). Furthermore, CKD may reduce the oncologist's clinical armamentarium as several chemotherapeutic agents are nephrotoxic and optimal surveillance imaging often requires iodinated contrast enhancement(16).

Regarding mortality, overall, approximately $26 \%$ of patients who were hospitalized died, and amongst hospi- 
talized cancer patients, approximately $34 \%$ of patients died. History of malignancy conferred a statistically significant $33 \%$ increased relative risk of mortality among hospitalized patients. However, upon analysis of malignancy sub-types, we found that this increased mortality risk appears to be driven by patients with pulmonary neoplasms. Indeed, patients with lung tumors had a greater than two-fold risk of death. On the contrary, patients with prostate, bladder, kidney, or general genitourinary malignancies were not at greater risk of mortality compared to the patients without cancer. Thus, though patients with malignancy may be at higher risk for mortality, this heightened risk does not appear to apply to patients with genitourinary malignancies. Interestingly, though patients with lung cancer and cancer in general had higher mortality rates, they did not have higher rates of ICU admission. This may be related to the shortage of ICU beds during the local peaks of the pandemic(23).

In addition to evaluating outcomes amongst hospitalized patients, we evaluated risk of hospitalization amongst all SARS-CoV-2 positive patients. Overall, we found no increased risk of hospitalization amongst patients with a history of cancer compared to those without a cancer diagnosis. Interestingly, we found an overall lower risk of hospitalization amongst patients with a history of genitourinary malignancy compared to patients without cancers. Indeed, on subgroup analysis this lower risk of hospitalization appears to be driven by patients with a history of kidney cancer. The explanation for this trend is unclear. However, one possibility is that the renin-angiotensin-system (RAS) is altered in patients with renal cancer. Indeed, SARS-CoV-2 binds via the angiotensin-converting enzyme 2 receptor(24) and previous research has shown that patients with renal cancer have differential expressions of RAS enzymes(25). Ultimately, further research is required to understand this pattern.

Our study has several notable limitations. We conducted a retrospective analysis of an existing database rather than a prospective study. Furthermore, given the nature of the database, we did not have data regarding tumor stage and grade and accordingly could not control for these variables. For example, in defining a patient as having a history of "lung cancer," we included all patients with a diagnosis code indicative of a lung neoplasm, including secondary and unspecified lung neoplasms. Given that the lung is a common site of metastatic spread, our lung cancer cohort may have included patients with more advanced malignancies which may in turn be contributing to the higher mortality rate observed. Indeed, patients also were not separated by those with active malignancy and those on surveillance. Additionally, amongst the individual genitourinary malignancies analyzed (prostate, bladder, kidney), our sample sizes were small. Despite these limitations, we believe our study provides an important adjunct to the existing literature.

\section{CONCLUSIONS}

Overall, our study findings suggest that patients with a history of cancer who are hospitalized with SARS$\mathrm{CoV}-2$ are not at greater risk for AKI, though they are at higher risk for mortality as compared to hospitalized patients without a cancer diagnosis. The increased risk in mortality appears driven by patients with pulmonary neoplasms. Patients with genitourinary malignancies do not appear to be at higher risk for mortality or for AKI compared to those without malignancy.

This finding is of great clinical importance as we navigate the "new-normal" phase of the COVID19 pandemic. Healthcare providers must guide patients through weighing the risks of delaying therapy against the risks of contracting COVID19 in the nosocomial setting. Understanding that the risk of adverse outcomes in patients with histories of genitourinary malignancy is similar to that of the general public may help guide patients through this challenging and anxiety provoking risk-benefit analysis. Further study with a larger cohort of genitourinary malignancy patients in a prospective manner is warranted.

\section{REFERENCES}

1. Wosik J, Fudim M, Cameron B, Gellad ZF, Cho A, Phinney D, et al. Telehealth transformation: COVID19 and the rise of virtual care. Journal of the American Medical Informatics Association. 2020;27(6):957-62.

2. Goldman HB, Haber GP. Recommendations for Tiered Stratification of Urological Surgery Urgency in the COVID-19 Era. Journal of Urology. 2020;204(1):11-3. 
3. Stensland KD, Morgan TM, Moinzadeh A, Lee CT, Briganti A, Catto JWF, et al. Considerations in the Triage of Urologic Surgeries During the COVID-19 Pandemic. European urology. 2020;77(6):663-6.

4. Wallis CJD, Novara G, Marandino L, Bex A, Kamat AM, Karnes RJ, et al. Risks from Deferring Treatment for Genitourinary Cancers: A Collaborative Review to Aid Triage and Management During the COVID-19 Pandemic. European urology. 2020;78(1):29-42.

5. Russell B, Liedberg F, Khan MS, Nair R, Thurairaja R, Malde S, et al. A Systematic Review and Metaanalysis of Delay in Radical Cystectomy and the Effect on Survival in Bladder Cancer Patients. European urology oncology. 2020;3(2):239-49.

6. van den Bergh RC, Albertsen PC, Bangma CH, Freedland SJ, Graefen M, Vickers A, et al. Timing of curative treatment for prostate cancer: a systematic review. European urology. 2013;64(2):204-15.

7. Berenguer J, Ryan P, Rodríguez-Baño J, Jarrín I, Carratalà J, Pachón J, et al. Characteristics and predictors of death among 4,035 consecutively hospitalized patients with COVID-19 in Spain. Clinical Microbiology and Infection. 2020.

8. Liang W, Guan W, Chen R, Wang W, Li J, Xu K, et al. Cancer patients in SARS-CoV-2 infection: a nationwide analysis in China. The Lancet Oncology. 2020;21(3):335-7.

9. Miyashita H, Mikami T, Chopra N, Yamada T, Chernyavsky S, Rizk D, et al. Do patients with cancer have a poorer prognosis of COVID-19? An experience in New York City. Annals of oncology : official journal of the European Society for Medical Oncology. 2020;31(8):1088-9.

10. Kuderer NM, Choueiri TK, Shah DP, Shyr Y, Rubinstein SM, Rivera DR, et al. Clinical impact of COVID19 on patients with cancer (CCC19): a cohort study. Lancet (London, England). 2020;395(10241):1907-18.

11. Lee A, Naksukpaiboon P. COVID-19 mortality in patients with cancer on chemotherapy or other anticancer treatments: a prospective cohort study. The Lancet. 2020;395.

12. Robilotti EV, Babady NE, Mead PA, Rolling T, Perez-Johnston R, Bernardes M, et al. Determinants of COVID-19 disease severity in patients with cancer. Nature Medicine. 2020;26(8):1218-23.

13. Chen Y-T, Shao S-C, Hsu C-K, Wu IW, Hung M-J, Chen Y-C. Incidence of acute kidney injury in COVID-19 infection: a systematic review and meta-analysis. Critical Care. 2020;24(1):346.

14. Yang X, Jin Y, Li R, Zhang Z, Sun R, Chen D. Prevalence and impact of acute renal impairment on COVID-19: a systematic review and meta-analysis. Critical Care. 2020;24(1):356.

15. Belayev LY, Palevsky PM. The link between acute kidney injury and chronic kidney disease. Current opinion in nephrology and hypertension. 2014;23(2):149-54.

16. Santos MLC, de Brito BB, da Silva FAF, Botelho A, de Melo FF. Nephrotoxicity in cancer treatment: An overview. World journal of clinical oncology. 2020;11(4):190-204.

17. Kumar RK, Sammon JD, Kaczmarek BF, Khalifeh A, Gorin MA, Sivarajan G, et al. Robot-assisted partial nephrectomy in patients with baseline chronic kidney disease: a multi-institutional propensity scorematched analysis. European urology. 2014;65(6):1205-10.

18. Ning C, Hu X, Liu F, Lin J, Zhang J, Wang Z, et al. Post-surgical outcomes of patients with chronic kidney disease and end stage renal disease undergoing radical prostatectomy: 10-year results from the US National Inpatient Sample. BMC nephrology. 2019;20(1):278.

19. Hamano I, Hatakeyama S, Iwamurau H, Fujita N, Fukushi K, Narita T, et al. Preoperative chronic kidney disease predicts poor oncological outcomes after radical cystectomy in patients with muscle-invasive bladder cancer. Oncotarget. 2017;8(37):61404-14.

20. Berlin DA, Gulick RM, Martinez FJ. Severe Covid-19. The New England journal of medicine. 2020. 
21. Giannakoulis VG, Papoutsi E, Siempos II. Effect of Cancer on Clinical Outcomes of Patients With COVID-19: A Meta-Analysis of Patient Data. JCO Global Oncology. 2020(6):799-808.

22. NYC Department of Health COVID19 Data Page [Available from: https://www1.nyc.gov/site/doh/covid/covid-19-data-archive.page.

23. Sprung CL, Joynt GM, Christian MD, Truog RD, Rello J, Nates JL. Adult ICU Triage During the Coronavirus Disease 2019 Pandemic: Who Will Live and Who Will Die? Recommendations to Improve Survival. Critical care medicine. 2020;48(8):1196-202.

24. Zhang H, Penninger JM, Li Y, Zhong N, Slutsky AS. Angiotensin-converting enzyme 2 (ACE2) as a SARS-CoV-2 receptor: molecular mechanisms and potential therapeutic target. Intensive Care Medicine. 2020;46(4):586-90.

25. Larrinaga G, Pérez I, Sanz B, Blanco L, López JI, Cándenas ML, et al. Angiotensin-converting enzymes (ACE and ACE2) are downregulated in renal tumors. Regulatory peptides. 2010;165(2-3):218-23.

\section{FIGURES}

Figure 1: Study Population and Cohort Definition

\section{Hosted file}

image1.emf available at https://authorea.com/users/359406/articles/481418-cancer-mortalityand-acute-kidney-injury-among-hospitalized-patients-with-sars-cov-2-infection

Tables

Table 1: Patient Characteristics, Overall and by Mortality among Hospitalized Patients

\begin{tabular}{llll}
\hline Characteristics & All $(\mathrm{N}=4018)$ & Died $(\mathrm{N}=1045)$ & Alive $(\mathrm{N}=2973)$ \\
\hline Any Cancer & $374(9 \%)$ & $126(12 \%)$ & $248(8 \%)$ \\
Kidney Cancer & $15(0.4 \%)$ & $4(0.4 \%)$ & $11(0.4 \%)$ \\
Prostate Cancer & $76(2 \%)$ & $29(2.8 \%)$ & $47(1.6 \%)$ \\
Bladder Cancer & $14(0.3 \%)$ & $5(0.5 \%)$ & $9(0.3 \%)$ \\
Lung Cancer & $14(0.3 \%)$ & $6(0.6 \%)$ & $38(1.2 \%)$ \\
Hematological Cancer & $54(1 \%)$ & $16(1.5 \%)$ & $67(2.3 \%)$ \\
Genitourinary Cancer & $106(2.6 \%)$ & $39(3.7 \%)$ & 61,17 \\
Age (mean, SD) & 64,17 & 73,12 & $1400(47 \%)$ \\
Women & $1844(46 \%)$ & $444(42 \%)$ & $750(25 \%)$ \\
Race & & & $767(26 \%)$ \\
White & $1000(25 \%)$ & $250(24 \%)$ & $1456(49 \%)$ \\
Black & $1060(26 \%)$ & $293(28 \%)$ & $1379(46 \%)$ \\
Other & $1958(49 \%)$ & $502(48 \%)$ & $721(24 \%)$ \\
Insurance Status & & $726(69 \%)$ & $873(29 \%)$ \\
Medicare & $2105(52 \%)$ & $157(15 \%)$ & \\
Medicaid & $878(22 \%)$ & $162(16 \%)$ & $2033(68 \%)$ \\
Other & $1035(26 \%)$ & $647(62 \%)$ & $759(26 \%)$ \\
Smoking Status & & $345(33 \%)$ & $181(6 \%)$ \\
Never & $2680(67 \%)$ & $53(5 \%)$ & $300(10 \%)$ \\
Current & $1104(27 \%)$ & $110(11 \%)$ & $774(26 \%)$ \\
Former & $234(6 \%)$ & $338(32 \%)$ & $1176(40 \%)$ \\
Obesity & $410(10 \%)$ & $523(50 \%)$ & \\
Diabetes & $1112(28 \%)$ & & \\
Hypertension & $1699(42 \%)$ & &
\end{tabular}




\begin{tabular}{llll}
\hline Characteristics & All $(\mathrm{N}=4018)$ & Died $(\mathrm{N}=1045)$ & Alive $(\mathrm{N}=2973)$ \\
\hline $\begin{array}{l}\text { Coronary Artery } \\
\text { Disease }\end{array}$ & $630(16 \%)$ & $222(21 \%)$ & $408(14 \%)$ \\
Atrial Fibrillation & $334(8 \%)$ & $133(13 \%)$ & $201(7 \%)$ \\
Chronic Kidney & $577(14 \%)$ & $195(19 \%)$ & $382(13 \%)$ \\
$\begin{array}{l}\text { Disease } \\
\text { Asthma }\end{array}$ & $257(6 \%)$ & $56(5 \%)$ & $201(7 \%)$ \\
$\begin{array}{l}\text { Chronic Obstructive } \\
\text { Pulmonary Disorder }\end{array}$ & $204(5 \%)$ & $74(7 \%)$ & $130(4 \%)$ \\
Acute Kidney Injury & $281(7 \%)$ & $103(10 \%)$ & $178(6 \%)$ \\
\hline
\end{tabular}

Table 2: Odds Ratios and 95\% Confidence Intervals from Multilevel Logistic Regression Model

\begin{tabular}{lllll}
\hline Comparison & Mortality & ICU & AKI & Hospitalization \\
\hline Cancer vs. No Cancer & $1.33(1.05,1.69)$ & $1.00(0.76,1.30)$ & $0.87(0.57,1.34)$ & $0.75(0.55,1.06)$ \\
Kidney Cancer vs. No Cancer & $0.65(0.21,2.08)$ & $0.61(0.13,2.73)$ & $0.54(0.07,4.01)$ & $0.41(0.18,0.95)$ \\
Kidney Cancer vs. Other Cancer & $0.55(0.16,1.95)$ & $0.58(0.12,2.85)$ & $0.79(0.10,5.98)$ & $0.83(0.35,1.97)$ \\
Prostate Cancer vs. No Cancer & $1.15(0.71,1.86)$ & $0.99(0.57,1.71)$ & $0.88(0.38,2.07)$ & $0.74(0.47,1.20)$ \\
Prostate Cancer vs. Other Cancer & $1.11(0.90,1.38)$ & $1.26(0.63,2.51)$ & $0.82(0.31,2.18)$ & $1.71(1.02,2.92)$ \\
Bladder Cancer vs. No Cancer & $0.87(0.29,2.65)$ & $0.66(0.14,2.99)$ & $* *$ & $0.50(0.19,1.32)$ \\
Bladder Cancer vs. Other Cancer & $0.88(0.27,2.92)$ & $0.75(0.16,3.55)$ & $* *$ & $1.20(0.45,3.24)$ \\
Lung Cancer vs. No Cancer & $2.44(1.05,4.39)$ & $0.99(0.27,3.60)$ & $1.16(0.15,8.69)$ & $0.85(0.54,1.28)$ \\
Lung Cancer vs. Other Cancer & $1.53(0.51,4.64)$ & $0.79(0.20,3.09)$ & $0.62(0.08,4.90)$ & $0.98(0.72,1.41)$ \\
Hematological Cancer vs. No Cancer & $1.32(0.72,2.43)$ & $1.18(0.62,2.24)$ & $0.98(0.34,2.78)$ & $1.01(0.65,1.78)$ \\
Hematological Cancer vs. Other Cancer & $1.14(0.82,1.57)$ & $1.03(0.77,1.50)$ & $0.92(0.41,1.83)$ & $1.10(0.68,1.96)$ \\
Genitourinary Cancer vs. No Cancer & $0.99(0.61,1.62)$ & $1.14(0.67,1.94)$ & $0.52(0.19,1.43)$ & $0.62(0.39,0.97)$ \\
Genitourinary Cancer vs. Other Cancer & $0.91(0.51,1.63)$ & $1.58(0.82,3.05)$ & $0.44(0.15,1.31)$ & $1.14(0.77,1.71)$ \\
\hline
\end{tabular}

**Not enough events to analyze

$\mathrm{AKI}=$ Acute Kidney Injury

$\mathrm{ICU}=$ intensive care unit admission 